\title{
Responses against islet antigens in NOD mice are prevented by tolerance to proinsulin but not IGRP
}

\author{
Balasubramanian Krishnamurthy, ${ }^{1}$ Nadine L. Dudek, ${ }^{1}$ Mark D. McKenzie, ${ }^{1}$ Anthony W. Purcell, ${ }^{2}$ \\ Andrew G. Brooks, ${ }^{3}$ Shane Gellert, ${ }^{4}$ Peter G. Colman, ${ }^{4}$ Leonard C. Harrison, ${ }^{5}$ Andrew M. Lew, ${ }^{5}$ \\ Helen E. Thomas, ${ }^{1}$ and Thomas W.H. Kay, ${ }^{1,6}$

\begin{abstract}
${ }^{1}$ St. Vincent's Institute, Fitzroy, Victoria, Australia. ${ }^{2}$ Department of Biochemistry and Molecular Biology, Bio21 Institute, and ${ }^{3}$ Department of Microbiology and Immunology, The University of Melbourne, Parkville, Victoria, Australia. ${ }^{4}$ Department of Diabetes and Endocrinology, The Royal Melbourne Hospital, Parkville, Victoria, Australia. ${ }^{5}$ The Walter and Eliza Hall Institute of Medical Research, Parkville, Victoria, Australia. ${ }^{6}$ Department of Medicine, St. Vincent's Hospital, The University of Melbourne, Fitzroy, Victoria, Australia.
\end{abstract}

\begin{abstract}
Type 1 diabetes (T1D) is characterized by immune responses against several autoantigens expressed in pancreatic $\beta$ cells. T cells specific for proinsulin and islet-specific glucose-6-phosphatase catalytic subunit-related protein (IGRP) can induce T1D in NOD mice. However, whether immune responses to multiple autoantigens are caused by spreading from one to another or whether they develop independently of each other is unknown. As cytotoxic T cells specific for IGRP were not detected in transgenic NOD mice tolerant to proinsulin, we determined that immune responses against proinsulin are necessary for IGRP-specific $T$ cells to develop. On the other hand, transgenic overexpression of IGRP resulted in loss of intra-islet IGRP-specific T cells but did not protect NOD mice from insulitis or T1D, providing direct evidence that the response against IGRP is downstream of the response to proinsulin. Our results suggest that pathogenic proinsulin-specific immunity in NOD mice subsequently spreads to other antigens such as IGRP.
\end{abstract}

\section{Introduction}

Type 1 diabetes (T1D) in humans and NOD mice results from $\mathrm{T}$ cell-mediated autoimmune destruction of insulin-producing $\beta$ cells of the pancreas (1). As T cells specific for a number of antigens have been recognized, it is easy to surmise that the disease is initiated by polyclonal activation of $\mathrm{T}$ cells to multiple specificities caused by a breakdown in normal tolerance to the $\beta$ cell. However, disease initiation may require $\mathrm{T}$ cells specific for 1 primary antigen; once the disease is initiated, $T$ cells specific for a number of additional antigens may develop by epitope spreading during the effector phase and mediate $\beta$ cell destruction. Two antigens implicated in the pathogenesis of T1D in NOD mice are proinsulin and islet-specific glucose-6-phosphatase catalytic subunit-related protein (IGRP). Cytotoxic T cells specific for either, derived without immunization from NOD mice, can transfer T1D (2-5). Whether responses to both or just one are required for T1D, and whether the responses are independent or related, remain unknown.

Several lines of evidence support the idea that initiation of autoimmunity in NOD mice is dependent on an immune response against insulin (6-8). Transgenic overexpression of proinsulin 2 in APCs of NOD mice (referred to here as NOD-PI mice) prevents insulitis and T1D. This may be due to enhanced negative selection of insulin-specific $\mathrm{T}$ cells, although the mechanism remains uncertain (6). Also, transfer of hematopoietic stem cells from NOD-PI mice prevents T1D in nontransgenic NOD recipient mice (7). Pro-

Nonstandard abbreviations used: IAA, insulin autoantibody; IGRP, islet-specific glucose-6-phosphatase catalytic subunit-related protein; ILN, inguinal lymph node; $\mathrm{m}-$, mouse; PLN, pancreatic lymph node; rh-, recombinant human; rm-, recombinant mouse; T1D, type 1 diabetes.

Conflict of interest: The authors have declared that no conflict of interest exists. Citation for this article: J. Clin. Invest. 116:3258-3265 (2006). doi:10.1172/JCI29602. tection from T1D in NOD-PI mice was confirmed in another study of NOD mice expressing mouse preproinsulin 2 under the control of an MHC class II invariant chain promoter (9). More recently, it was shown that proinsulin 1 and 2 knockout NOD mice are completely protected from development of anti-islet autoimmunity (8), although T1D is accelerated in NOD mice deficient in proinsulin 2 alone (10). While these data clearly implicate proinsulin in T1D induction, they do not exclude the possibility that other autoantigens have similar essential roles. The current study looks beyond the significant role for proinsulin to question the relationship between immune responses to proinsulin and other significant autoantigens. This is an important issue because in humans the development of autoimmune responses to multiple autoantigens makes progression to T1D much more likely and because the effectiveness of tolerance induction by specific antigens may be affected by the relationship between these immune responses.

Cells that recognize IGRP $206-214$ are present in the earliest islet infiltrates of NOD mice and undergo avidity maturation as islet inflammation progresses to overt disease $(11,12)$. TCR transgenic NOD mice have previously been generated using TCRs from the IGRP $_{206-214}$-reactive $8.3 \mathrm{~T}$ cell clone (4). These NOD8.3 TCR transgenic mice develop T1D at an accelerated rate $(4,13)$. Consistent with a role in disease progression, up to $1 \%$ of $\mathrm{CD}^{+} \mathrm{T}$ cells in peripheral blood and up to $30 \%$ of $\mathrm{CD} 8^{+} \mathrm{T}$ cells in the islets of nontransgenic NOD mice bind IGRP $206-214 \mathrm{H} 2-\mathrm{K}^{\mathrm{d}}$ tetramers (14). Interestingly, the number of these cells in the peripheral blood correlates with development of T1D in NOD mice (14).

The clonal dominance and pathogenicity of IGRP $206-214$-specific $\mathrm{T}$ cells in NOD mice suggest that these cells may play a crucial role in initiation or progression of T1D. To address whether they develop independently of $\mathrm{T}$ cells specific for proinsulin, we studied the fate of IGRP $206-214^{-}$-specific T cells in NOD-PI mice. To address 

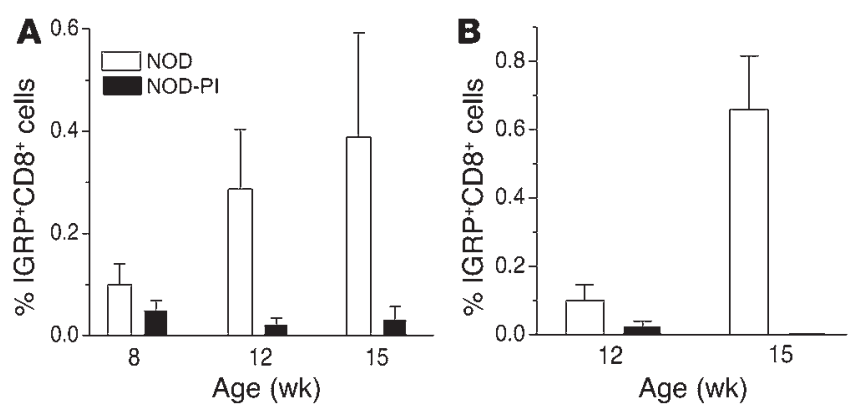

C

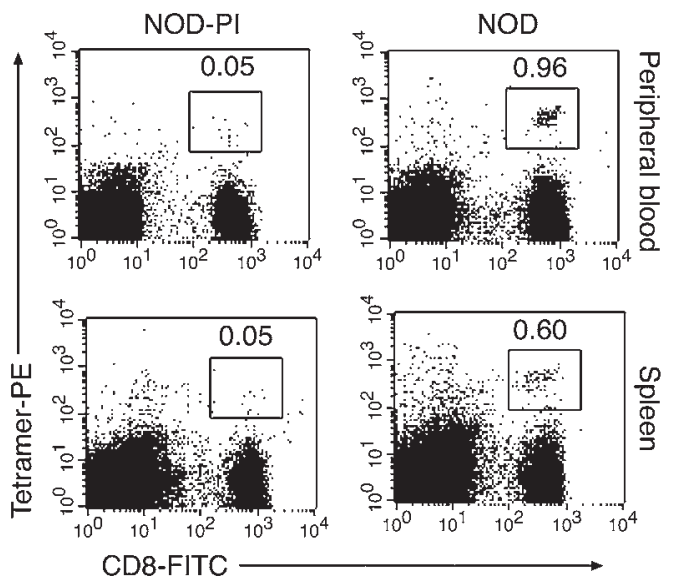

whether tolerance to IGRP prevents T1D, we studied how overexpression of IGRP under the control of MHC class II (I-E $\alpha$ ) promoter in NOD mice affected the incidence of insulitis and T1D. Understanding how the response to these autoantigens develops will help us to understand how antigen-specific tolerance induction could prevent T1D.

Our experiments suggest that immune response to IGRP I $_{26-214}$ does not develop in NOD-PI mice and NOD mice rendered tolerant to IGRP are not protected from insulitis and T1D.

\section{Results}

Lack of expansion of IGRP 206-214-specific T cells in NOD-PI mice. To assess whether tolerance to insulin affects the development of autoreactive $T$ cells specific for other antigens, the frequency of IGRP $_{206-214^{-}}$ specific T cells in NOD mice was compared with that observed in NOD-PI mice, which have minimal insulitis and do not progress to T1D (6). T cells that bind IGRP $206-214$ were readily detected in

\section{Figure 2}

T cell responses in NOD and NOD-PI mice after immunization with IGRP $206-214$ peptide. NOD and NOD-PI mice were primed with $25 \mu \mathrm{g}$ of IGRP $206-214$ peptide in CFA $(n=4)$ or with CFA alone $(n=2)$. After 1 week, splenocytes were collected and cultured with $10^{6} 1500$-cGy-irradiated IGRP $206-214$ loaded NOD splenocytes in complete RPMI supplemented with $10 \mathrm{U} / \mathrm{ml}$ rhlL-2 for 6 days. A sample of cultured cells was stained with IGRP $206-214$ tetramer and analyzed by flow cytometry and the remainder used as effectors in a cytotoxicity assay. (A) IGRP $206-214$ tetramer-positive CD8 ${ }^{+} \mathrm{T}$ cells from spleens of NOD and NOD-PI mice immunized with IGRP $206-214$ peptide in CFA or with CFA alone. (B) IGRP $206-214^{-}$specific CD8+ $\mathrm{T}$ cells from spleens of NOD-PI mice following priming with IGRP ${ }_{206-214}$ peptide in CFA were cytotoxic, as assessed by a standard 4 -hour ${ }^{51} \mathrm{Cr}$ release assay.

\section{Figure 1}

IGRP $_{206-214}$ tetramer-positive $\mathrm{CD}^{+} \mathrm{T}$ cell numbers in peripheral blood and spleens of NOD and NOD-PI mice. (A and B) Percentage of IGRP ${ }_{206-214}$-positive CD8+ T cells within (A) peripheral blood and (B) spleen. Data (mean \pm SEM) are from mice 8 weeks $(n=8)$, 12 weeks $(n=12)$ and 15 weeks of age $(n=12) . P<0.005$, NOD versus NOD-PI in peripheral blood at 12 weeks; $P<0.001$, NOD versus NOD-PI in peripheral blood at 15 weeks; $P<0.02$, NOD versus NOD-PI in spleen at 15 weeks. (C) Representative tetramer staining patterns. The numbers within dot plots indicate percentages of tetramer-positive CD8+ $\mathrm{T}$ cells.

NOD tissues and reached a peak at 15 weeks of age but few were detected in the peripheral blood or lymphoid tissues of NOD-PI mice at any age (Figure 1).

In NOD-PI mice there is no direct intervention against IGRP; hence the inability to detect $\mathrm{IGRP}_{206-214}$-specific $\mathrm{T}$ cells in NOD-PI mice may be due to the fact that they circulate at a low frequency. To test this, we primed 8-week-old NOD and NOD-PI mice with IGRP $206-214$ in CFA or with CFA alone. T cells were then expanded by culture with antigen for 8 days, and the proportion of IGRP $_{206-214^{-}}$-specific CD8 ${ }^{+}$ $\mathrm{T}$ cells was determined. IGRP $206-214$-specific $\mathrm{T}$ cells were detected in NOD-PI mice primed with IGRP $206-214$ in CFA (Figure 2A), indicating that $\mathrm{IGRP}_{206-214}$-specific $\mathrm{CD} 8^{+} \mathrm{T}$ cells were present at a low frequency and had not been deleted. IGRP $206-214$-specific $\mathrm{CD}^{+} \mathrm{T}$ cells induced by immunization were also observed in NOD mice.

It has previously been shown that progression to destructive insulitis and T1D in NOD mice is characterized by an increase in highavidity IGRP ${ }_{206-214}$-specific $\mathrm{T}$ cells (12). In contrast, in vitro studies suggest that low-avidity cells are significantly less cytotoxic (12). The expanded IGRP $206-214$-specific cells in immunized NOD-PI mice could represent high-affinity cytotoxic $\mathrm{T}$ cells or simply the

A
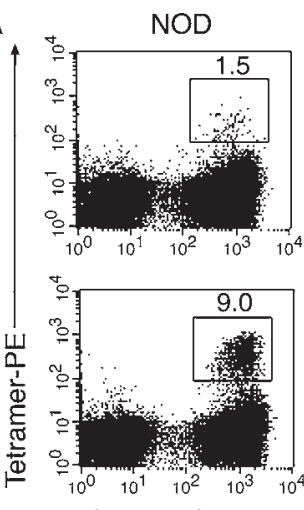

CD8-APC
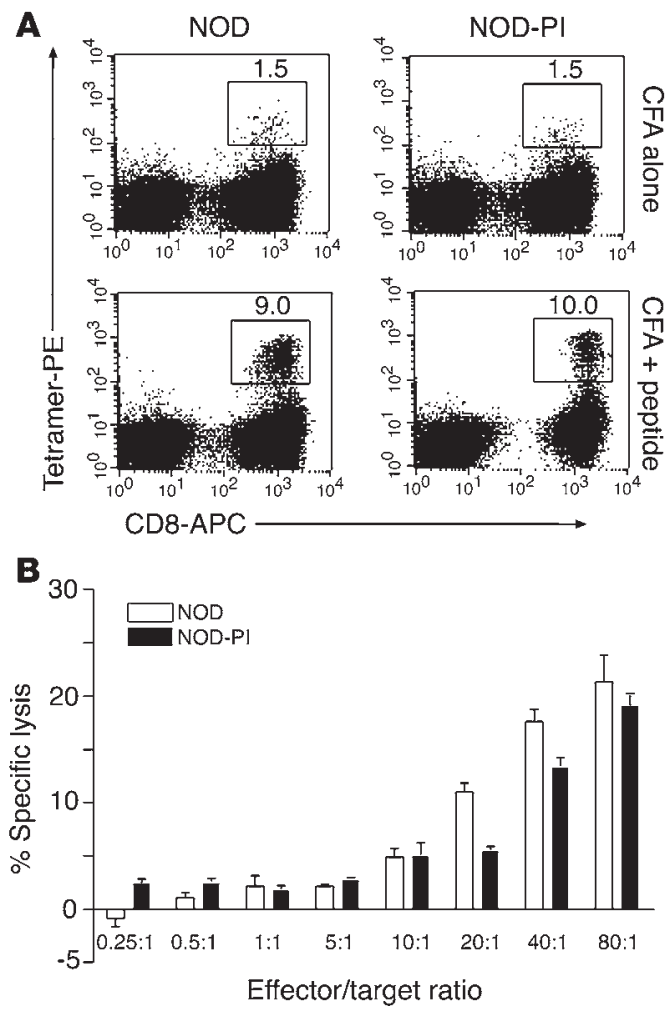
A
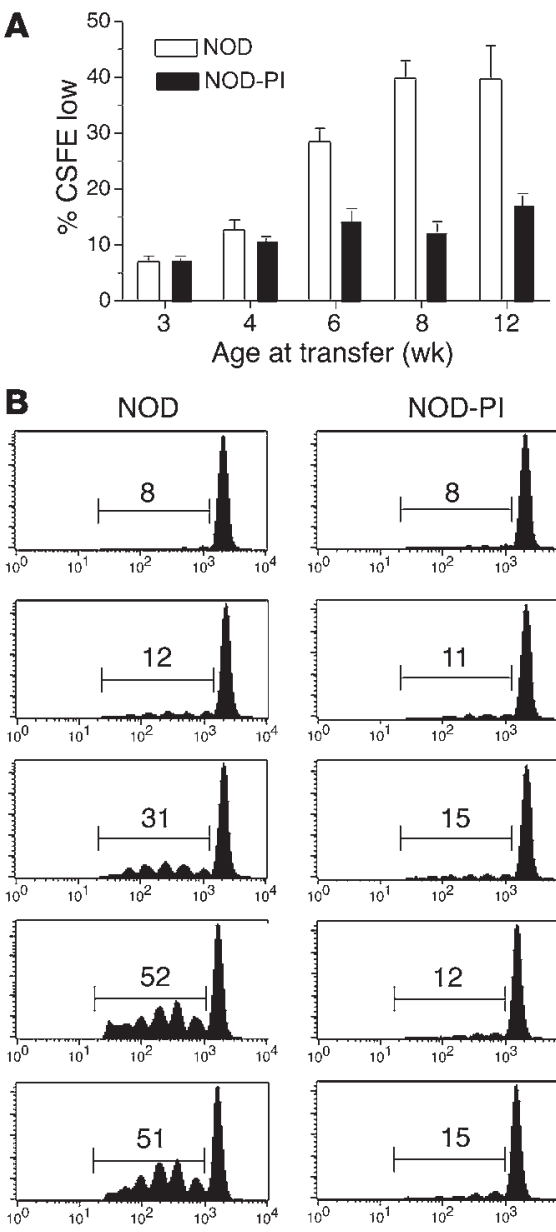

CFSE
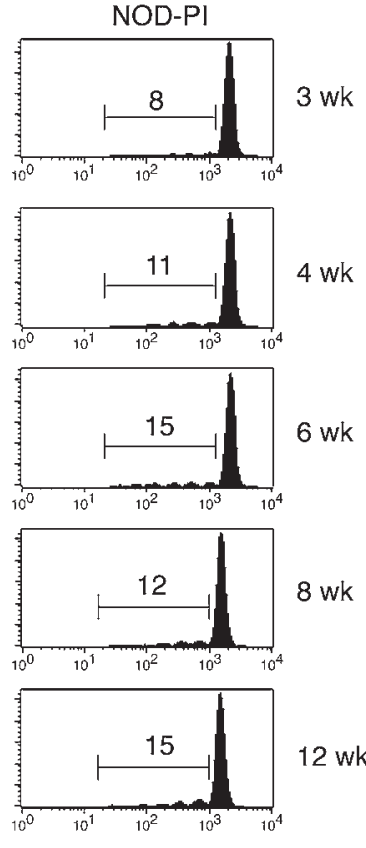

$12 \mathrm{wk}$

low-affinity $\mathrm{T}$ cells that remained after deletion of high-affinity cells. We tested the ability of the expanded IGRP ${ }_{206-214}$-specific $\mathrm{T}$ cells in NOD-PI mice to lyse ${ }^{51} \mathrm{Cr}$-loaded IGRP ${ }_{206-214}$ peptidepulsed P815 targets. We found the expanded IGRP $206-214$-specific $T$ cells from NOD-PI mice were able to kill the target cells, indicating that IGRP $206-214$-specific $\mathrm{T}$ cells from NOD-PI mice have cytotoxic potential but are "ignorant" of their antigen (Figure 2B).

Only a small percentage of $8.3 \mathrm{~T}$ cells proliferates in the pancreatic lymph nodes of NOD-PI mice. Cross-priming and activation of $\mathrm{CD}^{+} \mathrm{T}$ cells occurs when epithelial cell antigens are taken up and presented by DCs in draining lymph nodes "licensed" by inflammation or by activated $\mathrm{CD}^{+} \mathrm{T}$ cells. This is critical for initiation of T1D in experimental models (15). To determine whether IGRP ${ }_{206-214}$-specific $\mathrm{T}$ cells recognize cross-presented antigen in the pancreatic lymph nodes (PLNs) of NOD-PI mice, the proliferation of IGRP $206-214$-specific $\mathrm{CD}^{+} \mathrm{T}$ cells from NOD8.3 TCR transgenic mice was compared in NOD and NOD-PI hosts. We observed significantly lower proliferation of transferred cells in NOD-PI hosts as compared with NOD (Figure 3). DCs from spleen and bone marrow retained their ability to present antigen equally to the DCs from NOD mice, showing that transgene expression was not detrimental to APC function (Supplemental Figure 1, A and B; supplemental material available online with this article; doi:10.1172/JCI29602DS1). These findings are consistent with the view that the response to proinsulin contributes to increasing reactivity to IGRP.

\section{Figure 3}

Reduced proliferation of CFSE-labeled IGRP $206-214$-specific CD8+ T cells in NOD-PI mice. CFSE-labeled CD8 ${ }^{+} \mathrm{T}$ cells isolated from NOD8.3 TCR transgenic mice were injected $\left(4-6 \times 10^{6}\right.$ cells/mouse) intravenously into NOD and NOD-PI mice of different ages. Cells were isolated from PLN and ILN 3 days later and analyzed for CFSE dilution by flow cytometry. (A) Percentage (mean \pm SEM) of transferred cells dividing in NOD and NOD-PI mice ( $n=4-10$ mice per group at each time point). $P<0.004$, NOD versus NOD-PI in PLN at 6 weeks; $P<0.0002$, NOD versus NOD-PI in PLN at 8 weeks; $P<0.01$, NOD versus NOD-PI in PLN at 12 weeks. (B) Representative histogram plot. The numbers within the histogram plots indicate percentages of CFSE-low cells.

We looked for evidence of Tregs as an explanation for these findings. However, we - as other investigators previously found using these (6) or similar mice (9) - did not find any evidence for generation of Tregs by thymic expression of autoantigens. Splenocytes from NOD-PI mice did not differ from wild-type cells in their ability to modulate T1D induced by transfer of diabetic splenocytes. NOD8.3 T cells transferred into NOD mice proliferated to a similar extent whether cotransferred with $\mathrm{CD} 4^{+} \mathrm{CD} 25^{+}$or $\mathrm{CD} 4^{+} \mathrm{CD} 25^{-}$ cells from NOD-PI mice (Supplemental Figure 2A), and in vitro suppression of CD3-stimulated cells by $\mathrm{CD} 4{ }^{+} \mathrm{CD} 25^{+}$cells from NOD-PI mice was indistinguishable from that achieved using NOD Tregs (Supplemental Figure 2B).

IGRP expression in NOD-IGRP mice. The absence of responses to IGRP $_{206-214}$ in NOD-PI mice indicates that IGRP immune responses may be downstream of those to proinsulin. However, it is possible that this tolerance-induced protection from insulitis and T1D could be caused by other $\beta$ cell autoantigens, not just proinsulin. To determine whether this is the case, IGRP was expressed under the control of the MHC class II (I-E $\alpha \kappa)$ promoter in NOD mice (NOD-IGRP mice) exactly analogous to NOD-PI mice. Five independent NOD-IGRP transgenic lines were established. Expression of IGRP was observed in NOD-IGRP mice using real-time RT-PCR (Figure 4A).

To further confirm the expression of the IGRP transgene, we transferred CFSE-labeled IGRP $206-214$-specific T cells from NOD8.3 mice to NOD-IGRP mice and control littermates. As expected, in control littermates, transferred cells proliferated only in the draining PLNs. In contrast, extensive proliferation of the transferred cells in NOD-IGRP mice was noted in both the PLN and other lymph nodes, which was due to transgenic overexpression of IGRP in the APCs. This confirms that IGRP is expressed in the APCs of NOD-IGRP mice and that they remain capable of generating the 206-214 epitope presented to T cells in NOD mice (Figure 4B).

IGRP-specific tolerance. As IGRP $206-214$-specific T cells were detected at a high frequency in islets, lymphoid organs, and peripheral blood of NOD mice, we could test for tolerance to IGRP in unprimed NOD-IGRP mice. In contrast to control littermates, $T$ cells that bind IGRP $_{206-214}$ were not detected in the peripheral blood of NOD-IGRP mice (Figure 5). Furthermore, to assess the impact of transgenic IGRP expression on the specificity of islet-infiltrating T cells, isletinfiltrating $T$ cells from NOD-IGRP mice and control littermates were expanded by culturing individually selected islets in complete RPMI supplemented with recombinant human IL-2 (rhIL-2) for 7-9 days. The expanded $\mathrm{T}$ cells were stained with $\mathrm{IGRP}_{206-214} \mathrm{~K}^{\mathrm{d}}$ tetramers and analyzed by flow cytometry. There was a complete absence of IGRP $206-214$-specific cells in NOD-IGRP mice, whereas up to $40 \%$ of the islet-infiltrating $\mathrm{CD}^{+} \mathrm{T}$ cells in the control littermates 
A
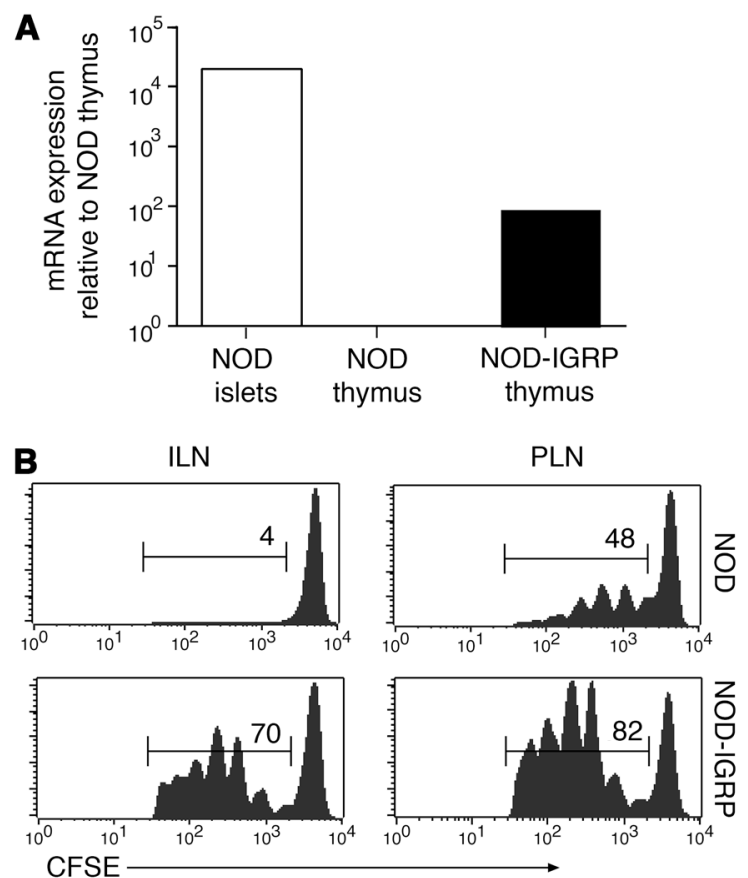

CFSE were specific for IGRP $_{206-214}$. These results were reproducible in 2 NOD-IGRP lines that were tested, consistent with tolerance to the IGRP $_{206-214}$ epitope in NOD-IGRP mice (Figure 6).

Spontaneous $\mathrm{CD} 4^{+} \mathrm{T}$ cell response to IGRP peptides have been reported in NOD mice (16). To test for tolerance to MHC class IIrestricted peptides in NOD-IGRP mice, we cultured splenocytes from 8-week-old NOD and NOD-IGRP mice in the presence of $100 \mu \mathrm{g} / \mathrm{ml}$ of IGRP $_{4-22}$ peptide. While spontaneous $\mathrm{CD}^{+} \mathrm{T}$ cell response was demonstrated in NOD mice, no response was detected in NOD-IGRP mice (Supplemental Figure 1C).

It was previously reported that immunizing NOD mice with IGRP 206-214 peptide did not prevent T1D despite near complete depletion of IGRP ${ }_{206-214}$-specific $\mathrm{T}$ cells because it was associated with an increase in responses against subdominant epitopes of IGRP (17). To test whether T1D in NOD-IGRP mice is due to expansion of $\mathrm{T}$ cells specific for subdominant epitopes of IGRP, we used T cells expanded from islets of NOD-IGRP or control NOD mice as effectors in a ${ }^{51} \mathrm{Cr}$ release assay against P815 loaded with either dominant IGRP $206-214$ or subdominant IGRP I1-29 $_{21}$ and IGRP 324-332 peptides. In contrast to $\mathrm{T}$ cells from NOD mice, $\mathrm{T}$ cells from NOD-IGRP mice did not kill targets loaded with either dominant or subdominant IGRP peptides (Figure 7A). These data show that NOD-IGRP mice demonstrate tolerance to both MHC class II- and MHC class I-restricted IGRP peptides. To confirm that $\mathrm{CD} 8^{+} \mathrm{T}$ cells other than those specific for IGRP are able to destroy $\beta$ cells during the effector phase of T1D, $\mathrm{T}$ cells were expanded from islets of nontransgenic NOD mice. They

\section{Figure 5}

IGRP $_{206-214}$ tetramer-positive CD8+ $\mathrm{T}$ cell numbers in peripheral blood of 12-week-old NOD and NOD-IGRP mice. (A) Percentage of IGRPpositive CD8 ${ }^{+} \mathrm{T}$ cells in peripheral blood $(n=14$ per group). $P<0.005$, NOD-IGRP versus controls. (B) Representative tetramer staining patterns. The numbers within the dot plots indicate percentages of tetramer-positive CD8 ${ }^{+} \mathrm{T}$ cells.

\section{Figure 4}

Expression of IGRP in NOD-IGRP mice. (A) Expression of transgenic IGRP mRNA in the thymi of NOD-IGRP or nontransgenic control littermates as compared with IGRP mRNA in islets of NOD mice. Total RNA from NOD-IGRP mice and nontransgenic control littermates was reverse transcribed using random primers. Real-time RT-PCR was performed with Assay-on-Demand kits for mouse IGRP and $\beta$-actin. (B) Functional IGRP expression in APCs of NOD-IGRP mice. CD8 ${ }^{+} \mathrm{T}$ cells from a NOD8.3 mouse were labeled with CFSE and transferred into 6-week-old NOD-IGRP or nontransgenic control littermates $(n=4$ per group). Recipients were sacrificed 3 days later, and their PLNs and ILNs were examined for $\mathrm{CFSE}^{+}$cells. The numbers within the histogram plots indicate percentages of CFSE-low cells.

were sorted by flow cytometry on the basis of whether they bound or did not bind $\mathrm{H}-2 \mathrm{~K}^{\mathrm{d}} \mathrm{IGRP}_{206-214}$ tetramer. Both tetramer-positive and tetramer-negative $\mathrm{T}$ cells killed $\beta$ cells from NOD mice in ${ }^{51} \mathrm{Cr}$ release assays (Figure $7 \mathrm{~B}$ ). To test whether $\mathrm{T}$ cells specific for proinsulin epitope $\mathrm{B}_{15-23}$ expand in the absence of IGRP ${ }_{206-214}$-specific $\mathrm{T}$ cells in NOD-IGRP mice, we used T cells expanded from islets of NOD-IGRP or control NOD mice as effectors in a ${ }^{51} \mathrm{Cr}$ release assay against P815 loaded with INS L9 peptide (LYLVCGERL; modified [Gly9Leu] from its endogenous counterpart, insulin $\mathrm{B}_{15-23}$, to increase MHC class I stability without affecting CTL binding ability) or IGRP $206-214$ peptide. Our results show T cells specific for insulin $\mathrm{B}_{15-23}$ peptide did not expand significantly in NOD-IGRP mice over NOD mice (Figure 7C).

To test immune responses against insulin, we measured insulin autoantibodies (IAAs) in 12-week-old NOD-IGRP, NOD-PI, and NOD mice. IAA responses were similar in NOD-IGRP and NOD mice. As expected, NOD-PI mice showed no IAA response (Figure 8).

Insulitis and T1D in NOD-IGRP mice. We investigated anti- $\beta$ cell immunity in NOD-IGRP mice to determine whether recognition of IGRP represents an important event for the development of autoimmunity. NOD-IGRP mice exhibited similar incidence and

A
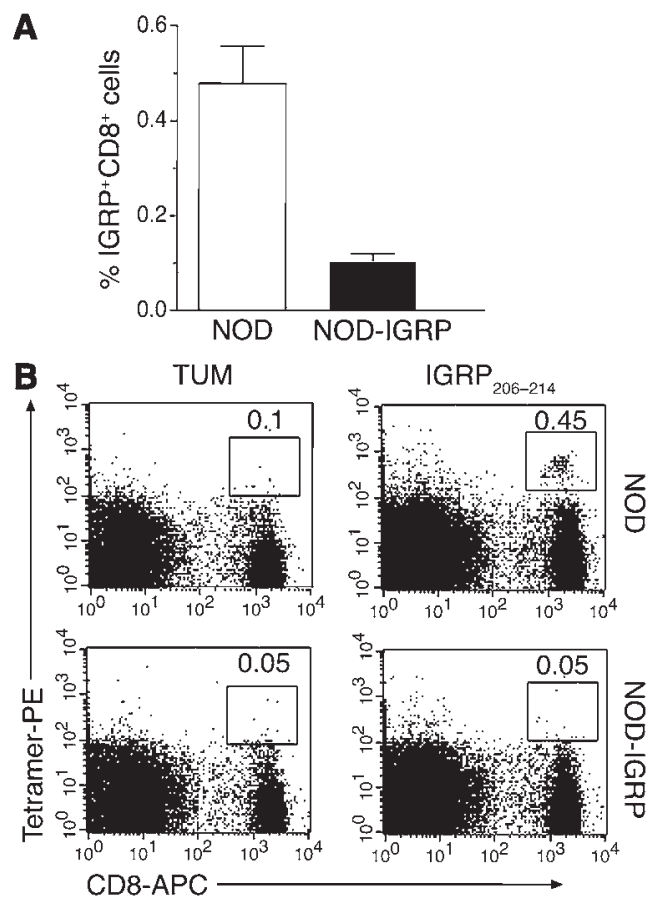




Figure 6

IGRP $_{206-214}$-specific CD8 ${ }^{+} \mathrm{T}$ cell response in NOD-IGRP mice. Isletinfiltrating $T$ cells from NOD-IGRP mice and nontransgenic control littermates were expanded by culturing individually selected islets in RPMI supplemented with $10 \mathrm{U} / \mathrm{ml}$ rhlL-2 for 7-9 days. The cultured T cells were stained with IGRP or TUM H-2K tetramers and analyzed by flow cytometry.

severity of insulitis as compared with nontransgenic littermates. These results were similar for 2 independent NOD-IGRP transgenic lines (Figure 9). NOD-IGRP mice developed T1D at a rate similar to NOD mice, indicating that while they can be pathogenic, IGRP-specific $\mathrm{T}$ cells are not essential for T1D.

\section{Discussion}

$\mathrm{T}$ cell reactivity to multiple antigens in the NOD mouse and multiple islet autoantibodies in humans may indicate parallel, unrelated targets contributing independently to disease pathogenesis or a primary initiating antigen that causes responses to other autoantigens once islet inflammation occurs. Proinsulin overexpressed in the thymus in NOD-PI mice prevented expansion of $\mathrm{CD}^{+} \mathrm{T}$ cells specific for the major epitope of IGRP, an antigen not directly targeted by the tolerance induction treatment. This result favors the interpretation that IGRP responses occur only once proinsulin responses have been established and that the immune response "spreads" from proinsulin to IGRP, although other interpretations are also possible. Accumulating evidence has established proinsulin as the primary autoantigen in T1D $(6,8,9)$. However, this is the first time to our knowledge that the relationship between proinsulin and IGRP responses has been directly explored. It is also what we believe to be the most direct evidence that the concept of spreading of autoimmune responses that is important in other

\section{Figure 7}

Cytotoxicity of islet-infiltrating $T$ cells as assessed by a standard 4-hour ${ }^{51} \mathrm{Cr}$ release assay. (A) T cells expanded from islets of NODIGRP mice $(n=4)$ or control littermates $(n=4)$ were assessed for cytotoxicity against P815 cells loaded with the indicated peptides. (B) T cells expanded from islets of nontransgenic NOD mice were sorted by flow cytometry on the basis of whether they bound or did not bind $\mathrm{H}-2 \mathrm{~K}^{\mathrm{d}}$ IGRP $\mathrm{F}_{206-214}$ tetramer. Both tetramer-positive and tetramer-negative T cells were able to kill $\beta$ cells from NOD mice. (C) T cells expanded from islets of NOD-IGRP $(n=5)$ or NOD mice $(n=8)$ were assessed for cytotoxicity against P815 cells loaded with indicated peptides. diseases, particularly diseases induced by immunization with autoantigens (18), applies to the spontaneous development of T1D in the NOD mouse. Interestingly, the requirement for responses to proinsulin was even present in NOD8.3 mice, which have a preexisting repertoire of IGRP $206-214$-reactive cells. These mice have dramatically reduced T1D when crossed with NOD-PI mice (B. Krishnamurthy and T.W.H. Kay, unpublished observations). $\mathrm{CD}^{+} \mathrm{T}$ cells are necessary for T1D to fully develop in NOD8.3 mice; we speculate that proinsulin-specific $\mathrm{CD} 4^{+} \mathrm{T}$ cells help NOD8. 3 cells to become activated and pathogenic.

The lack of deletion of $\mathrm{IGRP}_{206-214^{-}}$-specific $\mathrm{CD}^{+} \mathrm{T}$ cells and reduced NOD8.3 T cell proliferation observed in the PLNs of NOD-PI mice are consistent with $T$ cell ignorance of IGRP. T cells specific for IGRP escaped deletion in the thymus, were not tolerant, and were activated by appropriate stimulation. In NOD mice, IGRP was presented in the PLN in a way that enabled activation, expansion, migration to the pancreatic islets, and $\beta$ cell destruction. In NOD-PI mice and in non-T1D-prone strains, presentation of IGRP was reduced and was insufficient for $\mathrm{T}$ cell expansion to occur. IGRP $206-214$-specific $T$ cells remained present at low frequencies but were expanded by immunization, raising the possibility that the tolerance to IGRP in NOD-PI mice could potentially be broken.

A further test of the hypothesis that proinsulin is upstream of IGRP and plays a more primary role in the disease process was to determine the effects of IGRP expression in APCs. Unlike NOD-PI mice, NOD-IGRP mice developed IAAs and insulitis similar to nontransgenic control littermates, but without IGRP-specific $\mathrm{CD}^{+} \mathrm{T}$ cells in the insulitis lesion. This confirmed that the response to proinsulin, but not IGRP, is required for initiation of autoimmunity. Interestingly, transgenic expression of GAD65 did not prevent T1D in NOD mice, although the interpretation of this was less clear because the pathogenic significance of autoimmunity to GAD65 is less well established than for IGRP (19). Autoantigens in T1D, a spontaneous autoimmune disease, may fall into categories of initiating and downstream antigens, as is the case in autoimmune diseases induced by exogenous antigens such as EAE (20). Once autoimmunity is initiated by insulin-specific $\mathrm{T}$ cells, $\mathrm{T}$ cells specific for several other antigens that include but are not restricted to IGRP may develop and may be important in mediating $\beta$ cell destruction. During this phase the status of CD8 responses
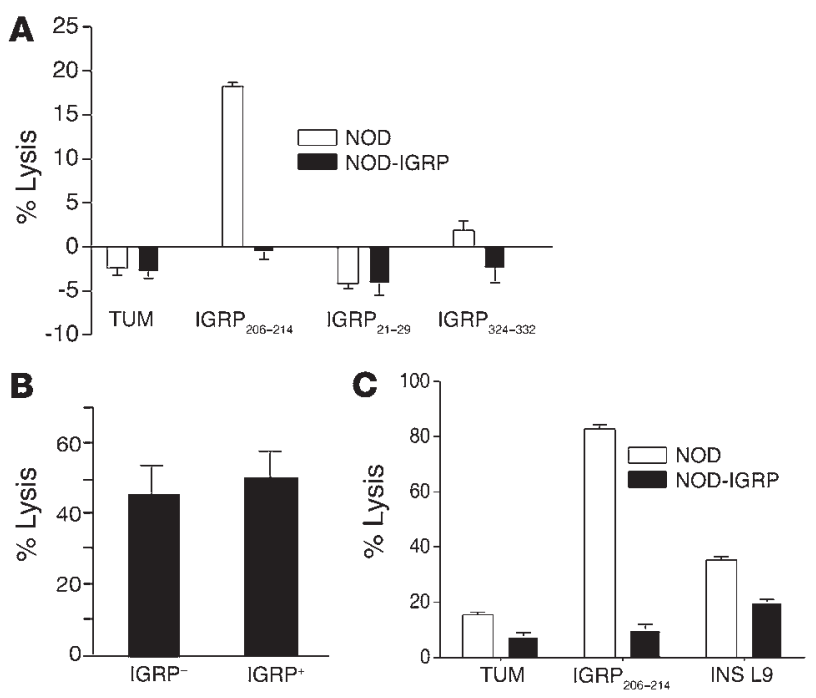


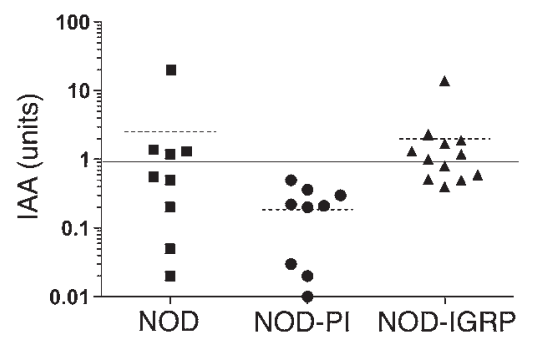

Figure 8

Spontaneous anti-insulin response. IAAs in 12-week-old female NOD $(n=10)$, NOD-PI $(n=10)$, and NOD-IGRP $(n=13)$ mice. Horizontal line indicates upper limit of normal for IAAs. Dotted lines indicate mean for each group. $P<0.03$, NOD versus NOD-PI; $P=\mathrm{NS}$, NOD versus NOD-IGRP.

against proinsulin may still be important; however, the response to the $\mathrm{B}_{15-23}$ epitope of proinsulin has waned, having been dominant in very early insulitis (2). Our data suggests that the responses to this epitope do not expand in NOD-IGRP mice. It is also possible that $\mathrm{T}$ cells specific for many antigens develop simultaneously, and not one after the other as we have proposed. Proinsulin-reactive $\mathrm{T}$ cells would then be essential to drive the expansion of IGRPreactive $T$ cells (and $T$ cells reactive to other antigens). In this situation, proinsulin-reactive $T$ cells remain the primary driver cells, even though they may have developed at the same time.

The evidence that IGRP responses occurred only once islet immune reactivity was established questions whether IGRP responses are truly pathogenic or are simply a reflection of the release of $\beta$ cell antigens. It has long been debated whether autoimmunity is sometimes the result of rather than the cause of target cell damage. There is, however, strong evidence that IGRP $_{206-214^{-}}$ specific $T$ cells are important during the effector phase of the disease. Progression of insulitis to T1D in NOD mice was accompanied by cyclic expansion of the circulating IGRP $206-214$-specific $\mathrm{T}$ cell pool and by avidity maturation of its islet-associated counterpart. The NY8.3 clone specific for IGRP $206-214$ was isolated from newly diabetic NOD mice and was potently able to cause disease. Our data showed progression to T1D in NOD-IGRP mice despite apparent lack of CD4 and CD8 responses to IGRP. It is possible that residual $\mathrm{T}$ cell responses to IGRP not measured by the assays we used persist in these mice, and absolute proof about the role of IGRP in progression to T1D in NOD mice will require analysis of a knockout of IGRP on the NOD background.

It is plausible that the proposed relationship between proinsulin (an important antigen early in the pathogenesis of T1D in NOD mice) and other antigens such as IGRP and GAD (more important in the effector phase) is preserved in humans. IGRP itself has not been shown to be a significant antigen in human T1D to date, but the concept of initiating and effector antigens may apply in human T1D. Serological studies of young at-risk children indicate that autoimmunity to insulin generally precedes that to GAD65 or IA-2/phogrin $(21,22)$, consistent with a role for insulin as an initiator antigen, whereas antibodies to IA-2 often emerge closer to diagnosis. At the level of the individual, however, any of these 3 autoantibodies may appear first, and in older subjects IAAs are detected only in a minority of patients at disease onset. It is also possible that there is no universal primary or initiating autoantigen in humans, but rather that disease is initiated by differ- ent autoantigens in different individuals. Progression to T1D is faster in individuals with multiple autoantibodies. The presence of multiple autoantibodies has been interpreted as meaning that the autoantibody responses are true rather than false positives, i.e., that the connection drawn between multiple autoantibodies and progression to T1D is due to diagnostic test performance. Our data, however, suggest that the acquisition of immune responses against multiple autoantigens is a necessary step in pathogenesis and that the reduced risk of a single autoantibody reflects failure of the immune response to spread. This is an important distinction. The effect of tolerance to proinsulin on immune responses to other antigens is a relevant issue in clinical trials of T1D prevention in humans. Our findings suggest that primary prevention should be most effective if targeted at initiating antigens such as proinsulin. Once insulitis is established, tolerance induction to other $\beta$ cell antigens will need to be considered.

\section{Methods}

Mice. NOD-PI mice, NOD mice expressing mouse proinsulin 2 under the control of an MHC class II (I-E $\alpha$ K) promoter, and NOD8.3 mice, NOD mice expressing the TCR $\alpha \beta$ rearrangements of the $\mathrm{H}-2 \mathrm{~K}^{\mathrm{d}}$-restricted, $\beta$ cell-reactive $\mathrm{CD}^{+} \mathrm{T}$ cell clone NY8.3, have been described previously $(4,6)$. NOD-PI mice were used after breeding to homozygosity. All animal studies were carried out at St. Vincent's Institute in accordance with accepted standards of humane animal care and were approved by the animal ethics committee of St. Vincent's Health (Melbourne, Australia).

Generation of transgenic NOD-IGRP mice. A 1,068-bp fragment encompassing the protein coding region of mouse IGRP was amplified by PCR using NIT-1 insulinoma cell line cDNA as template. The PCR product was subcloned into the PGEM $T$ easy vector, verified by sequencing, and excised
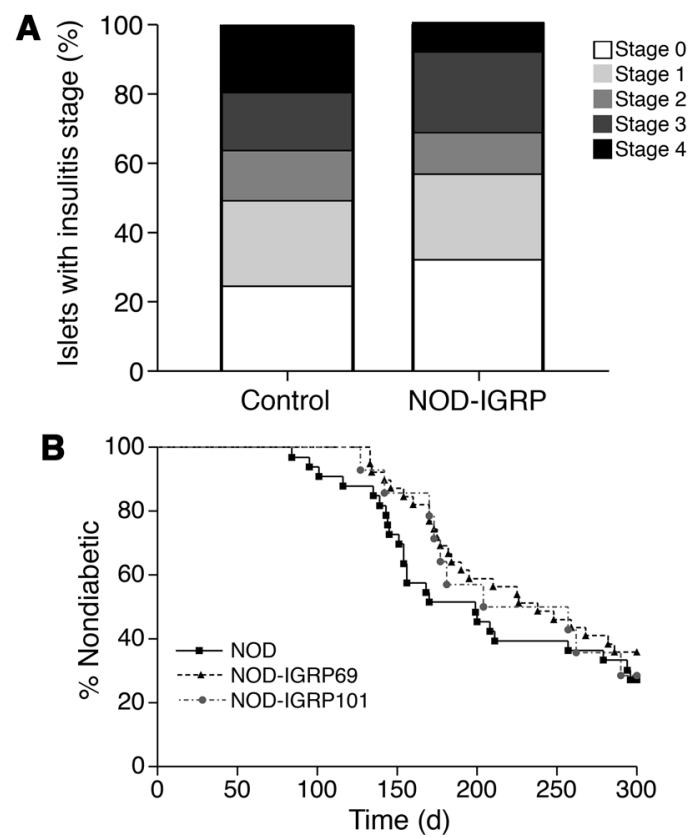

\section{Figure 9}

Insulitis and T1D in NOD-IGRP versus control mice. (A) Histological grading of insulitis in pancreas sections of 100-day-old mice $(n=8$ per group). $P=$ NS, NOD-IGRP versus controls. (B) Incidence of T1D in NOD-IGRP mice (founder lines NOD-IGRP 69, $n=39$; and NOD-IGRP $101, n=18)$ and control littermates $(n=33)$. $P=\mathrm{NS}$, NOD-IGRP versus controls. 
between HindIII and Pst-I. This, along with a 1.8-kb fragment encoding the 5 ' flanking sequence nucleotides $-1,903$ to -39 of the MHC class II (I-EoK) promoter and a $650-\mathrm{bp}$ fragment encoding polyadenylation signal, was cloned into bluescript KS-. The transgene fragment between $B s s H I I$ sites was purified. The purified transgene construct was injected into fertilized NOD ova and reimplanted into foster mothers using standard techniques in the transgenic mouse facility at the Walter and Eliza Hall Institute. Offspring were screened by PCR of genomic DNA using 2 sets of transgenespecific primers, the first spanning MHC class II (I-E $\alpha$ ) promoter and IGRP (forward, 5'-TAGGACCTGGTTGCAAGGAA-3'; reverse, 5'-AGGACGATGGCCAAACAATA-3') and the second spanning introns 2 and 3 within the IGRP gene (forward, 5'-TGCGTCTGGTATGTCATGGT-3'; reverse, $5^{\prime}$-TGCATTGTGGTCAGTGAGGT-3').

Expression analysis. Total RNA was prepared from frozen tissue samples using TRIzol reagent (Invitrogen). Total RNA ( $1 \mu \mathrm{g})$ was reverse transcribed using random primers. Real-time RT-PCR analysis was performed with Assay-on-Demand kits (Applied Biosystems) for mouse IGRP (mIGRP) and $\beta$-actin (housekeeping gene). Analysis was performed on a Rotor-Gene-3000 (Corbett Research; Corbett Life Science).

Peptides and tetramers. The peptides IGRP ${ }_{206-214}$ (VYLKTNVFL), IGRP ${ }_{4-22}$ (LHRSGVLIIHHLQEDYRTY), IGRP 22-29 (TYYGFLNFM), IGRP ${ }_{324-332}$ (SFCKSASIP), INS L9 (LYLVCGERL), and TUM (KYQAVTTTL) were synthesized by Auspep. H-2 $\mathrm{K}^{\mathrm{d}}$ tetramers were made by ImmunoID. Tetramer specificity was validated by staining spleen cells from NOD8.3 mice.

Tetramer staining. Islet-infiltrating $\mathrm{T}$ cells were expanded by culturing individually selected islets in RPMI (Invitrogen) supplemented with antibiotics, $2 \mathrm{mM}$ glutamine, nonessential amino acids, $50 \mu \mathrm{M}$ mercaptoethanol, $10 \%$ FCS (complete RPMI), and $10 \mathrm{U} / \mathrm{ml}$ rhIL-2 for 6-9 days. The cultured $\mathrm{T}$ cells from islets or single-cell suspensions from peripheral blood, spleen, and PLNs were stained with $0.5 \mu \mathrm{g} \mathrm{IGRP}_{206-214}$ or TUM H- $2 \mathrm{~K}^{\mathrm{d}}$ tetramers for 3 hours on ice in PBS/2\% FCS, then with FITC-conjugated anti-CD8 (clone 53-6.7; BD Biosciences - Pharmingen) and PerCP-conjugated antiB220 (clone RA3-6B2; BD Biosciences - Pharmingen) for 30 minutes, all on ice. Stained cells were analyzed by flow cytometry (FACSCalibur; BD). Tetramer positivity was determined using a lymphocyte gate and exclusion of $\mathrm{B} 220^{+}$cells. Tetramer-positive cells are expressed as percentage of $\mathrm{CD}^{+}{ }^{+} \mathrm{B} 220^{-}$cells minus the percentage of TUM tetramer-positive cells. Data were analyzed using CellQuest software (version 5.1.1; BD).

CFSE labeling and adoptive transfer. $\mathrm{CD}^{+} \mathrm{T}$ cells from NOD8.3 mice were purified using magnetic beads (Miltenyi Biotec). T cells were resuspended in PBS containing 0.1\% BSA (Sigma-Aldrich) at $10^{7}$ cells $/ \mathrm{ml}$. For labeling, $1 \mu \mathrm{l}$ of a CFSE (Invitrogen) stock solution ( $5 \mathrm{mM}$ in DMSO) was incubated with $10^{7}$ cells for 10 minutes at $37^{\circ} \mathrm{C}$. After incubation, cells were washed 3 times with RPMI supplemented with $5 \%$ FCS. In each experiment, $5 \times 10^{6}$ cells in $200 \mu \mathrm{l}$ PBS were injected intravenously into the tail veins of NOD or NOD-PI hosts. Hosts were sacrificed 3 days later, and their PLNs and inguinal lymph nodes (ILNs) were examined for $\mathrm{CFSE}^{+}$cells.

Preparation of DCs. Bone marrow-derived DCs were prepared by culturing bone marrow cells in the presence of recombinant mouse GM-CSF (rmGM-CSF) and rmIL-4 (5 ng/ml) for 8 days as described previously (23). Splenic DC isolation was performed as described by Vremec et al. (24). Spleen fragments were digested for 20 minutes at room temperature with collagenase-DNase and then treated for 5 minutes with EDTA to disrupt T cell-DC complexes. Light density cells were enriched by centrifugation on a $1.077 \mathrm{~g} / \mathrm{cm}^{3}$ Nycodenz layer (Nycomed) for 10 minutes at $1,700 \mathrm{~g}$. NonDCs were depleted by incubating in optimized concentrations of $\mathrm{mAbs}$ (anti-CD3 [KT3.1.1], anti-Thy1 [T24/31.7], anti-B220 [RA36B2], anti-GR-1 [RB68C5], and anti-erythrocyte [TER119]) followed by removal of the Abbinding cells with anti-rat Ig-coupled magnetic beads (Dynabeads; Dynal). The resulting DCs were greater than $70 \% \mathrm{CD} 11 \mathrm{c}^{+}$and $\mathrm{I}-\mathrm{Ag}^{\mathrm{7}}+$.
In vitro T cell proliferation assay. Splenic $8.3 \mathrm{CD}^{+} \mathrm{T}$ cells $\left(2 \times 10^{4}\right.$ cells/well $)$ were incubated in triplicate with $\operatorname{IGRP}_{206-214^{-}}(0.1 \mu \mathrm{M})$ or TUM-pulsed $(0.1$ $\mu \mathrm{M})$ bone marrow- or spleen-derived DCs $\left(10^{4} \mathrm{cells} /\right.$ well $)$. The cells were cultured in 96-well round-bottomed plates in $200 \mu \mathrm{l}$ complete RPMI (complete RPMI) for 3 days at $37^{\circ} \mathrm{C}$ in $5 \% \mathrm{CO}_{2}$. Each well was pulsed with $1 \mu \mathrm{Ci}$ of $\left[{ }^{3} \mathrm{H}\right]$-thymidine during the last 18 hours of culture. For spontaneous $\mathrm{CD} 4^{+}$ response, splenic T cells from NOD and NOD-IGRP mice were cultured in the presence or absence of $100 \mu \mathrm{g} / \mathrm{ml} \mathrm{IGRP}_{4-22}$ peptide as described previously (16). For inhibition assay, sorted $\mathrm{CD} 4^{+} \mathrm{CD} 25^{-} \mathrm{T}$ cells $\left(2 \times 10^{4}\right)$ from the spleens of NOD mice were cultured with varying numbers of sorted $\mathrm{CD} 4^{+}$ $\mathrm{CD} 25^{+} \mathrm{T}$ cells from the spleens of NOD-PI or NOD mice in the presence of $10^{4} \mathrm{NOD}$ spleen-derived DCs and antibody to CD3 (clone 2C11;5 $\mu \mathrm{g} / \mathrm{ml}$ ).

Generation of IGRP-specific CTL. Mice were primed with $25 \mu \mathrm{g}$ IGRP $_{206-214}$ peptide in CFA (Sigma-Aldrich). After 1 week, regional lymph nodes were collected and cultured with $10^{6}$ 1,500 cGy-irradiated IGRP 206-214-loaded NOD splenocytes in complete RPMI supplemented with $10 \mathrm{U} / \mathrm{ml}$ rhIL-2 for 6 days. Cytotoxicity was assessed by standard ${ }^{51} \mathrm{Cr}$ release assay using P815 cells as targets.

${ }^{51} \mathrm{Cr}$ release assay. Whole islets or cells of the $\mathrm{H}-2^{\mathrm{d}}$ mastocytoma line P815 were loaded with $150 \mu \mathrm{Ci}\left[{ }^{51} \mathrm{Cr}\right]$ sodium chromate (Amersham Biosciences) for 90 minutes, washed, and resuspended in complete RPMI. P815 cells $\left(5 \times 10^{3}\right.$ cells/well $)$ loaded with indicated peptide or islets ( 10 per well) were incubated with $\mathrm{T}$ cells in triplicate for $4-16$ hours at $37^{\circ} \mathrm{C}$ in round-bottomed 96-well plates. The radioactivity of the harvested supernatants was measured in a Cobra gamma counter (PerkinElmer).

Histological scoring and diabetes incidence. Pancreata were dissected, snapfrozen in OCT (Miles-Yeda) in a hexane/liquid nitrogen bath, and stored at $-70^{\circ} \mathrm{C}$. Serial $5-\mu \mathrm{m}$ cryostat sections were cut, fixed in acetone, and air dried. Immunohistochemical staining for insulin was performed as described previously (25). Islets were scored as follows: 0 , no lesions; 1 , peri-islet infiltrates; $2,<25 \%$ islet destruction; $3,>25 \%$ islet destruction; 4 , complete islet destruction. Mice were monitored for diabetes by weekly measurement of urinary glucose levels using Diastix (Bayer). Those suspected of hyperglycemia were confirmed by measurement of blood glucose levels using ACCU-CHEK Advantage II strips (Roche Diagnostics). Mice with blood glucose levels greater than $15 \mathrm{mM}$ were considered diabetic.

IAA assay. IAAs were measured with a 96-well filtration plate micro-IAA assay as described previously (26).

Statistics. Data were analyzed using 1-way ANOVA with Bonferroni's post test for comparison of multiple columns. Cumulative incidence of diabetes was determined by Kaplan-Meier estimates, and statistical analysis of difference was determined by log-rank test. A value of $P<0.05$ was considered significant.

\section{Acknowledgments}

We thank Bill Heath and Jim McCluskey for helpful comments on the manuscript, Pere Santamaria for the NOD8.3 mice, Linus Chang for help with tetramer production, and Lina Mariana and Rochelle Ayala-Perez for technical assistance. This work was supported by a Program Grant from the National Health and Medical Research Council of Australia, a Center Grant from the Juvenile Diabetes Research Foundation (JDRF), and postdoctoral fellowships from the JDRF (to B. Krishnamurthy and H.E. Thomas) as well as by a Millennium Grant of Diabetes Australia (to T.W.H. Kay).

Received for publication July 5, 2006, and accepted in revised form October 3, 2006.

Address correspondence to: Thomas W.H. Kay, St. Vincent's Institute, 41 Victoria Parade, Fitzroy, Victoria 3065, Australia. Phone: 61-3-9288-2480; Fax: 61-3-9416-2676; E-mail: tkay@svi.edu.au. 
1. Tisch, R., and McDevitt, H. 1996. Insulin-dependent diabetes mellitus. Cell. 85:291-297.

2. Wong, F.S., et al. 1999. Identification of an MHC class I-restricted autoantigen in type 1 diabetes by screening an organ-specific cDNA library. Nat. Med. 5:1026-1031.

3. Wong, F.S., Visintin, I., Wen, L., Flavell, R.A., and Janeway, C.A., Jr. 1996. CD8 T cell clones from young nonobese diabetic (NOD) islets can transfer rapid onset of diabetes in NOD mice in the absence of CD4 cells. J. Exp. Med. 183:67-76.

4. Verdaguer, J., et al. 1997. Spontaneous autoimmune diabetes in monoclonal $\mathrm{T}$ cell nonobese diabetic mice. J. Exp. Med. 186:1663-1676.

5. Lieberman, S.M., et al. 2003. Identification of the beta cell antigen targeted by a prevalent population of pathogenic CD8 + T cells in autoimmune diabetes. Proc. Natl. Acad. Sci. U. S. A. 100:8384-8388.

6. French, M.B., et al. 1997. Transgenic expression of mouse proinsulin II prevents diabetes in nonobese diabetic mice. Diabetes. 46:34-39.

7. Steptoe, R.J., Ritchie, J.M., and Harrison, L.C. 2003. Transfer of hematopoietic stem cells encoding autoantigen prevents autoimmune diabetes. J. Clin. Invest. 111:1357-1363. doi:10.1172/ JCI200315995.

8. Nakayama, M., et al. 2005. Prime role for an insulin epitope in the development of type 1 diabetes in NOD mice. Nature. 435:220-223.

9. Jaeckel, E., Lipes, M.A., and von Boehmer, H. 2004. Recessive tolerance to preproinsulin 2 reduces but does not abolish type 1 diabetes. Nat. Immunol. 5:1028-1035.

10. Thebault-Baumont, K., et al. 2003. Acceleration of type 1 diabetes mellitus in proinsulin 2-deficient
NOD mice. J. Clin. Invest. 111:851-857. doi:10.1172/ JCI200316584.

11. DiLorenzo, T.P., et al. 1998. Major histocompatibility complex class I-restricted $T$ cells are required for all but the end stages of diabetes development in nonobese diabetic mice and use a prevalent $\mathrm{T}$ cell receptor alpha chain gene rearrangement. Proc. Natl. Acad. Sci. U. S. A. 95:12538-12543.

12. Amrani, A., et al. 2000. Progression of autoimmune diabetes driven by avidity maturation of a T-cell population. Nature. 406:739-742.

13. Verdaguer, J., et al. 1996. Acceleration of spontaneous diabetes in TCR-beta-transgenic nonobese diabetic mice by beta-cell cytotoxic CD $8+T$ cells expressing identical endogenous TCR-alpha chains. J. Immunol. 157:4726-4735.

14. Trudeau, J.D., et al. 2003. Prediction of spontaneous autoimmune diabetes in NOD mice by quantification of autoreactive $\mathrm{T}$ cells in peripheral blood. J. Clin. Invest. 111:217-223. doi:10.1172/JCI200316409.

15. Heath, W.R., et al. 2004. Cross-presentation, dendritic cell subsets, and the generation of immunity to cellular antigens. Immunol. Rev. 199:9-26.

16. Mukherjee, R., Wagar, D., Stephens, T.A., Lee-Chan, E., and Singh, B. 2005. Identification of CD4+ T cell-specific epitopes of islet-specific glucose-6phosphatase catalytic subunit-related protein: a novel beta cell autoantigen in type 1 diabetes. J. Immunol. 174:5306-5315.

17. Han, B., et al. 2005. Prevention of diabetes by manipulation of anti-IGRP autoimmunity: high efficiency of a low-affinity peptide. Nat. Med. 11:645-652.

18. Vanderlugt, C.L., and Miller, S.D. 2002. Epitope spreading in immune-mediated diseases: impli- cations for immunotherapy. Nat. Rev. Immunol. 2:85-95.

19. Jaeckel, E., Klein, L., Martin-Orozco, N., and von Boehmer, H. 2003. Normal incidence of diabetes in NOD mice tolerant to glutamic acid decarboxylase. J. Exp. Med. 197:1635-1644.

20. McMahon, E.J., Bailey, S.L., Castenada, C.V., Waldner, H., and Miller, S.D. 2005. Epitope spreading initiates in the CNS in two mouse models of multiple sclerosis. Nat. Med. 11:335-339.

21. Colman, P.G., et al. 2000. Islet autoimmunity in infants with a Type I diabetic relative is common but is frequently restricted to one autoantibody. Diabetologia. 43:203-209.

22. Ziegler, A.G., Hummel, M., Schenker, M., and Bonifacio, E. 1999. Autoantibody appearance and risk for development of childhood diabetes in offspring of parents with type 1 diabetes: the 2-year analysis of the German BABYDIAB Study. Diabetes. 48:460-468.

23. Son, Y.I., et al. 2002. A novel bulk-culture method for generating mature dendritic cells from mouse bone marrow cells. J. Immunol. Methods. 262:145-157.

24. Vremec, D., et al. 1992. The surface phenotype of dendritic cells purified from mouse thymus and spleen: investigation of the CD8 expression by a subpopulation of dendritic cells. J. Exp. Med. 176:47-58.

25. Thomas, H.E., Parker, J.L., Schreiber, R.D., and Kay, T.W. 1998. IFN- $\gamma$ action on pancreatic $\beta$ cells causes class I MHC upregulation but not diabetes. J. Clin. Invest. 102:1249-1257.

26. Williams, A.J., Bingley, P.J., Bonifacio, E., Palmer, J.P., and Gale, E.A. 1997. A novel micro-assay for insulin autoantibodies. J. Autoimmun. 10:473-478. 